\title{
PLANNING OF CHEMICAL SYNTHESIS BASED ON MONTE-CARLO TREE SEARCH
}

\section{A. Fatykhova', T. Madzhidov', R. Nugmanov' and A. Varnek ${ }^{2}$}

${ }^{1}$ Department of organic and medicinal chemistry, Kazan Federal University, 420000, Russia, Kazan, Kremlyovskaya, 18.

${ }^{2}$ Laboratory of chemoinformatics, University of Strasbourg, 67000, France, rue Blaise Pascal, 4.

DOI: 10.19163/MedChemRussia2021-2021-174

E-mail: adafatyhova@kpfu.ru

Currently, the planning of the chemical synthesis of any compound, in particular, medicinal compounds, is an extremely important task. Modern methods of drug development, such as virtual screening, docking, de novo design, allow the generation of many molecules with the necessary biological activity and other properties. One of the problems is that some of the promising molecules cannot move to the next stage of development due to the problem of their synthesis. In this work, we present an approach to planning forward chemical synthesis (from reagents to products) based on the Monte Carlo tree search method. In contrast to the traditional retrosynthetic approach, the forward synthesis method makes it possible to predict the path of synthesis not only of the target molecule, but also of molecules similar to the target one. This is due to the fact that gradually approaching the target molecule, at each stage, the algorithm predicts many compounds that could potentially have similar properties.

The developed tool consists of several main blocks: a database of molecules and reaction rules, modules for virtual reactions generation, and heuristic algorithms for fast search based on similarity metrics. The developed approach uses commercially available chemical compounds as initial reagents and rules of reaction transformations to generate new products. Generation of reactions proceed using the Virtual Reactor [1], which allows the generation of chemically correct structures. Monte Carlo tree search methods allow you to effectively navigate the vast space of chemical compounds, finding the best solutions in the optimal time.

Acknowledgements. This work was supported by the Russian Science Foundation (19-73-10137).

\section{References}

[1] Nugmanov, R. et al. J. Chem. Inf. Model.2019 59 (6), 2516-2521. doi: 10.1021/ acs.jcim.9b00102. 